\title{
La Pauvrete Et Les Pauvres Dans La Ville De Bukavu. Essai D’ecriture Du Sens Et De La Causalite
}

\author{
Par Amos Cishunguluka Kanani \\ Bolinda Wa Bolinda
}

Le premier est enseignant et chercheur

à l'Université officielle de Bukavu à la Faculté de Sciences sociales, le second, à l'Université de Kisangani, en RD Congo

\section{doi: 10.19044/esj.2016.v13n2p305 URL:http://dx.doi.org/10.19044/esj.2016.v13n2p305}

\begin{abstract}
This article attempts to present the current image of poverty in order to establish its causality in Bukavu town. The populations interviewed did not have the same understanding of the spectrum of poverty, but know the external manifestations of poverty and the people considered or who consider themselves as being poor.

In addition, the survey has shown that poverty is an accumulation of deprivations or lacks; but monetary deprivation or lack of money was more prevalent in $11 \%$ of the opinions in all communes as meaning poverty. The enigma remains to know the amount of money you need to consider yourself or be considered poor or not. According to this research, although several causes or factors contribute to poverty in the city of Bukavu, bad governance at all levels is the most evoked factor and orchestrated by endogenous and exogenous, state and non-state actors.
\end{abstract}

Keywords: Poverty, poor, causality, actor, inequality

\section{Résumé}

Cet article tente de présenter le visage actuel de la pauvreté en vue d'établir sa causalité dans la ville de Bukavu.

Les populations enquêtées n'ont pas la même compréhension du spectre de la pauvreté, mais connaissent les manifestations extérieures par lesquelles sont décrites la pauvreté et les personnes considérées ou qui se considèrent comme des pauvres.

Bien plus, l'enquête a renseigné que la pauvreté est un cumul de privations ou de manques ; mais la privation monétaire ou le manque d'argent a été plus brandi par $11 \%$ d'opinion dans toutes les communes comme signifiant la pauvreté. L’énigme reste de savoir le montant d'argent qu’il faut posséder 
pour se considérer ou être considéré comme pauvre ou pas. Selon cette recherche, bien que plusieurs causes ou facteurs contribuent à la pauvreté dans la ville de Bukavu, la mauvaise gouvernance à tous les niveaux est le facteur le plus évoqué et orchestré par des acteurs endogènes et exogènes, étatiques et non étatiques.

Mots clés : Pauvreté, pauvre, causalité, acteur, inégalité

\section{Introduction}

\section{Le problème et les présupposés de la recherche}

L'un des ennemis les plus redoutables pour l'humanité se trouve être la pauvreté. Son histoire est liée à celle de l'homme, mais son sens, ses causes/ facteurs et les critères de distinction entre riches et pauvres diffèrent d'une société à une autre.

Diagnostiquer des situations de pauvreté dans une société n'est pas toujours facile, il subsiste le risque de se tromper; surtout si on n'interroge pas les différentes catégories sociales qui vivent les réalités qu'elles considèrent ou qu'on considère comme étant celles de pauvreté ou de non pauvreté.

Pour décrire les situations de pauvreté et estimer le nombre des pauvres, les indicateurs monétaires sont les plus couramment brandis. La Banque mondiale qui diffuse ces indicateurs internationaux admet pourtant la multi dimensionnalité de la pauvreté et soutient que les progrès sociaux ont longtemps été considérés comme une des composantes seulement de la croissance économique, et donc, sortir de la pauvreté passe exclusivement par un accroissement des revenus (Banque mondiale, 2000-2001, p. 6).

Le progrès social que cette institution subordonne à la croissance économique est défini par trois critères: la capacité d'une société à satisfaire les besoins de base de ses citoyens (qualité et accès au système de santé, au logement, à la sécurité...), à construire les fondements de leur bien-être sur la durée (en leur donnant accès à l'éducation et à l'information) et à leur donner l'opportunité de s'accomplir(Kirpalani Anita, avril 2013, p.13).

La RDC est rangée parmi les pays pauvres au monde et cela, malgré ses potentialités naturelles. L'Etat est considéré comme étant en faillite. Le PNUD a établi les taux de pauvreté par province et constate que la province du Sud-Kivu dont la ville de Bukavu qui constitue notre terrain de recherche est le chef-lieu, avait un taux de $84,7 \%$ et un taux de chômage le plus élevé du pays en 2013(22,2\%). Ce taux de pauvreté le hisse à la troisième position des provinces pauvres, après la province de l'Equateur $(93,6)$ et celle de Bandundu avec 89,1\% (PNUD, 2013, p.1 carte en pdf).

Dans la ville de Bukavu, les plaintes, les situations qui reflètent la misère, les attitudes et les sentiments d'infériorité et d'inutilité se 
développent dans les discours de plusieurs personnes. Mais, en même temps, on assiste à l'émergence des organisations de la société civile, parmi lesquelles les coopératives d'épargne et de crédit, initiées, semble-t-il, pour essayer d'aider les pauvres à vaincre la pauvreté.

Des hommes et des femmes s'inquiètent de leur existence, ils jugent très difficiles leurs conditions de vie, il s'observe une véritable compétition pour la survie. Pour un seul poste d'emploi déclaré disponible dans une ONG, des centaines des demandeurs vont se présenter. On ne saurait dire qu'il existe un seul secteur où cette lutte pour la survie ne s'observe pas dans la ville de Bukavu.

Pendant ce temps, plusieurs personnes ont adhéré massivement aux coopératives d'épargne et de crédit qui leur ont prêté de l'argent et facilité l'épargne, deux actions considérées comme motrices pour se libérer de la pauvreté. La plupart des bénéficiaires de ces crédits s'en sont servi pour initier des activités commerciales.

$\mathrm{Au}$ fil du temps, le retard de remboursement et l'insolvabilité commençaient à se faire constater. Dès lors, un climat délétère s'est installé, la confiance entre les coopératives et les membres bénéficiaires des crédits s'est effritée, obligeant soit de ralentir le rythme d'octroi des crédits soit de mettre en quarantaine les épargnes des membres, etc. On est passé de l'euphorie à la confrontation.

Les phénomènes de pauvreté s'observent encore dans cette ville, au point de laisser penser que la pauvreté est et reste une réalité du passé et du présent dans cette entité urbaine.

De ce qui précède, le présent article se propose de comprendre la pauvreté en tant que concept et réalité dans la ville de Bukavu. Il s'agit donc de repérer la signification de la pauvreté et du pauvre, et spécifiquement, identifier les causes/ facteurs qui alimentent la pauvreté ainsi que ses acteurs.

Hypothétiquement, pour la population de la ville de Bukavu, la pauvreté correspondrait au manque permanant d'argent et d'opportunités pour y accéder. Cet état de dénuement serait lié à plusieurs facteurs endogènes et exogènes imbriqués.

\section{Méthodologie}

La réalisation de cette étude a été ponctuée par deux moments clé : la collecte des données et le traitement/analyse des résultats.

$>\quad$ La collecte des données: A ce stade, nous avons mobilisé les techniques suivantes :

- L'observation, par laquelle les manifestations extérieures de la pauvreté en termes d'indicateurs ont été répertoriées; tels qu'ils sont identifiés dans le corps de ce papier. 
- $\quad$ Le questionnaire : cet outil de collecte des données était constitué de questions centrées sur les thèmes suivants: Existence de la pauvreté à Bukavu, sens donné à la pauvreté, causes/facteurs de la pauvreté, et acteurs de la pauvreté à Bukavu.

- L’échantillonnage : cette technique a opéré de la manière ci-après :

a) Ciblage des sites de l'enquête : les trois communes de la ville de Bukavu (Kadutu, Ibanda et Bagira) ont été retenues comme aires géographiques de l'enquête.

b) Détermination de l'échantillon :

- $\quad$ Dans chaque commune, nous avons distribué des questionnaires d'enquête aux personnes adultes sélectionnées de manière casuelle ou accidentelle. La collecte des données a pris fin lorsque nous avons constaté de la saturation des réponses des enquêtés.

Nous avons interrogé plus de personnes dans les communes de Kadutu et Bagira que dans celle d'Ibanda; pour la simple raison qu'à Bukavu, c'est dans ces deux communes qu'on pense qu'il existe plus de pauvres que dans celle d'Ibanda. Ainsi, la distribution du questionnaire a fait état de 336 personnesayant effectivement remis les fiches d'enquête contenant des réponses : 131 enquêtés dans la commune de Kadutu, 116 dans la commune de Bagira, et 89 dans celle d’Ibanda. Ces personnes étaient constituées de 162 hommes (48,2\%) et 174 femmes (51,8\%).

\section{$>\quad$ Le traitement des données/ analyse des résultats}

A ce stade, les données recueillies ont été d'abord encodées dans Excel puis analysées au moyen du logiciel SPSS 16.0. Cependant, d’autres données qualitatives ont été traitées sans logiciel.

Dans sa structuration, cet article traite de deux points saillants, hormis l'introduction et la conclusion. Le premier point aborde la pauvreté en tant que réalité à Bukavu, et le second est consacré à la question de la causalité et des acteurs de la pauvreté à Bukavu.

\section{La pauvrete en tant que realite a bukavu : ses manifestations et son sens}

Il s’agit dans ce chapitre de dresser le portrait de la pauvreté en tant que réalité dans la ville de Bukavu et en identifier les indicateurs ainsi que la signification.

\section{Portrait et manifestations de la pauvreté à Bukavu}

Tableau 1. Existence de la pauvreté

\begin{tabular}{|l|l|l|l|l|} 
& & Frequency & Percent & Valid Percent \\
\hline Valid & Non & 7 & 2.1 & 2.1 \\
& Oui & 329 & 97.9 & 97.9 \\
\cline { 2 - 5 } & Total & 336 & 100.0 & 100.0 \\
\hline
\end{tabular}


Les résultats visualisés dans ce tableau permettent de confirmer la présence de la pauvreté dans la ville de Bukavu. Près de $98 \%$ d'opinions émises affirment son existence. La pauvreté est donc vécue et connue des habitants de cette ville.

Lorsqu'on croise la variable commune et l'opinion sur l'existence de la pauvreté, on obtient le résultat présenté dans le tableau ci-dessous :

Tableau 02. Commune et existence de la pauvreté à Bukavu

\begin{tabular}{|c|c|c|c|c|}
\hline \multirow{2}{*}{\multicolumn{2}{|c|}{ Commune }} & \multicolumn{2}{c|}{ Existence de la pauvreté } & Total \\
\hline \multirow{2}{*}{ Bagira } & Oui & Non & \\
& \% within commune & 100 & 0 & 80 \\
\hline \multirow{2}{*}{ Ibanda } & Count & 119 &, $0 \%$ & $100,0 \%$ \\
& \% within commune & $96,7 \%$ & 4 & 123 \\
\hline \multirow{2}{*}{ Kadutu } & Count & 129 & $3,3 \%$ & $100,0 \%$ \\
\cline { 2 - 5 } & \% within commune & $97 \%$ & 4 & 133 \\
\hline Total & Count & 328 & $8 \%$ & $100,0 \%$ \\
& \% within commune & $97,5 \%$ & $2,5 \%$ & $100,0 \%$ \\
\hline
\end{tabular}

L'existence de la pauvreté est évidemment confirmée dans chacune de trois communes de la ville de Bukavu, mais beaucoup plus dans la commune de Bagira où 100\% des enquêtés ont répondu affirmativement à la question de savoir si la pauvreté existe dans la ville de Bukavu. L'opinion affirmative représente globalement $97,5 \%$ tandis que celle négative représente 2,5\%. Cette pauvreté à Bukavu est connue par ses signes extérieurs sous forme des manifestations ou indicateurs (qualitatifs).

- $\quad$ Signes extérieurs (manifestations de la pauvreté) à Bukavu

- $\quad$ Chômage de masse et touchant plus les diplômés d'université ;

- Délinquance ou déviance sociale (vol simple et à main armée, prostitution, vagabondage sexuel, escroquerie, ...) ;

- $\quad$ Logement indécent pour plusieurs habitants et promiscuité (nombre des chambres insuffisant par rapport aux membres de ménages, logement en état de délabrement et situés sur des sites non viables ;

- $\quad$ Prédications et demandes d'aumône dans la rue et dans des espaces publics (marchés, écoles,...) ;

- $\quad$ Abandon scolaire à tous les cycles d'étude (primaire, secondaire, supérieur) ;

- $\quad$ Manque d'accès aux soins de santé (ce qui oblige certains à recourir à l'automédication, à la médecine traditionnelle tenue par des charlatans et à la prière) ;

- $\quad$ Propos de jalousie et de haine à l'égard de ceux qui ont une vie jugée heureuse ;

- $\quad$ Revenus salariaux faibles ;

- $\quad$ Développement du secteur informel (une petite économie marchande informelle); considéré par ses praticiens comme relevant de la 
« débrouille »;

- $\quad$ Présence d’enfants dans la rue ;

- $\quad$ Augmentation du nombre des mendiants qui sillonnent tous les artères de la ville pour demander de l'aide ;

- $\quad$ Escroquerie ;

- $\quad$ Habitations délabrées dans des quartiers populeux ;

- $\quad$ Constructions anarchiques et à des sites impropres ;

- $\quad$ prolifération du petit commerce à des endroits non autorisés (des marchés pirates) ;

- Faible accès aux services de base (électricité, eau) obligeant l'accordement clandestin ou illicite pour échapper aux factures ;

- $\quad$ Conditions d'hygiènes non réunies (insalubrité)

- $\quad$ Beaucoup de commerçants colporteurs (ambulants) vendant même dans les édifices publics ;

- $\quad$ Endettement faisant l'objet d'accusation devant la justice ;

- $\quad$ Prostitution et vagabondage sexuel ;

- $\quad$ Multiplicité des églises et des chambres de prière et des prédicateurs dans la rue pour pousser les gens à offrir l'aumône ;

- $\quad$ Les gens comptent plus sur la chance et dépensent de l'argent pour prétendre gagner au centuple par les jeux de hasard dit «tombola ou libaku » en swahili du milieu) et signifiant la chance en Français. C’est la recherche de l'argent facile ; gagner aux moindres efforts ;

- $\quad$ Insécurité liée au banditisme (ravir les biens aux passants et tuer par couteau dans des quartiers populeux aux heures plus ou moins tardives) ;

- Accusations sorcières et discours sur l'enrichissement par voie de fétichisme ; etc.

Ces mêmes personnes qui confirment que la pauvreté existe dans la ville de Bukavu et qui en ont décrit les manifestations, ont du même coup dégagé différents sens ou perceptions de la pauvreté.

\section{Qu'est-ce que la pauvreté pour la population à Bukavu ?}

Tableau 3. Sens donné à la pauvreté dans la ville de Bukavu

\begin{tabular}{|c|c|c|c|}
\hline $\mathbf{N}^{\circ}$ & Sens proposées de la pauvreté dans la ville de Bukavu & Effectif & Pourcentage \\
\hline 1 & Manque d'argent ou des moyens financiers de subsistance & 37 & 11 \\
\hline 2 & $\begin{array}{c}\text { Manque d'emploi pour les personnes actives et compétentes } \\
\text { (revenus) }\end{array}$ & 28 & 8,3 \\
\hline 3 & Manger mal et une seule fois par jour & 23 & 7 \\
\hline 4 & L'analphabétisme & 16 & 5 \\
\hline 5 & Manque de relations au niveau de la société & 21 & 6,2 \\
\hline 6 & Travailler sans être bien rémunérée & 20 & 6 \\
\hline 7 & Vivre dans un logement indécent & 20 & 6 \\
\hline 8 & Incapacité de supporter les coûts de soins médicaux & 19 & 6 \\
\hline 9 & Vivre dans l'insécurité permanente & 19 & 6 \\
\hline
\end{tabular}




\begin{tabular}{|c|c|c|c|}
\hline 10 & Etre incapable de résoudre seul ses problèmes & 18 & 5,3 \\
\hline 11 & Manque d'accès à l'eau potable et au courant électrique & 18 & 5,3 \\
\hline 12 & $\begin{array}{c}\text { Avoir plusieurs enfants alors qu'on n'a pas beaucoup } \\
\text { d'argent }\end{array}$ & 16 & 5 \\
\hline 13 & $\begin{array}{c}\text { Incapacité pour les parents de supporter la scolarité des } \\
\text { enfants }\end{array}$ & 16 & 5 \\
\hline 14 & Etat permanent de maladie ou une santé précaire & 15 & 4,4 \\
\hline 15 & Manque de parcelle et de maison en ville & 14 & 4,1 \\
\hline 16 & Manque de moyen de transport personnel & 14 & 4,1 \\
\hline 17 & Manque de champs, d'élevage ou d'unités de production & 13 & 4 \\
\hline 18 & Manque d'habits & 11 & 3,2 \\
\hline & TOTAL & 336 & 100 \\
\hline
\end{tabular}

Il n'y a pas de doute que pour la population de la ville de Bukavu, la pauvreté est un mal qui combine des handicaps de natures différentes. Elle est la résultante de plusieurs situations de manque ou de dénuement. Cependant, de tous ces manques, celui de l'argent au sens monétaire a été plus évoqué par les enquêtés, à l'ordre de $11 \%$ de l'échantillon. Le sens qui talonne ce premier semble en être l'explication (Pourquoi les gens manquent de l'argent). Il va de soi que le manque d'emploi alors qu'on a la force et la compétence expose inéluctablement au manque de revenus. Tant il est vrai que sans activité rémunératrice il n’y a pas de revenus, il restera aussi vrai que sans les revenus, pas de souveraineté.

Outre le manque d'argent comme entendement principal de la pauvreté à Bukavu, remarquons que les enquêtés ont également considéré que le manque de relations peut aussi appauvrir ; c'est la capital social qui est évoqué ici. Nous estimons que l'argent peut y contribuer tout comme les relations peuvent aussi procurer l'argent. De ce fait, il existerait un rapport d'inter influence entre l'argent et les relations sociales. Il s'observe que les populations possèdent leurs définitions de la pauvreté qui sont plus appropriées à leur existence passée, présente et future et souvent plus dynamiques (DSCRP, 4mai 2016).

Au niveau macro sociétal, l'entendement économico-financier de la pauvreté est le plus évoqué aussi. Le rapport de 2005 sur le diagnostic de la pauvreté en RDC présente les résultats de l'Analyse Participative de la pauvreté (APP) réalisée au cours de l'Atelier de Kisantu et fait ressortir un total de 61 sens que les congolais donnent à la pauvreté. Le rapport en retient trente sens considérés comme les principaux constituant $88 \%$ des réponses fournies par les répondants (Diagnostic de la pauvreté en rdc/rapport-2005, pp. 29-30).

Ainsi, selon l'ordre d'importance (sur base de la fréquence de chaque sens donné à la pauvreté), les pauvres en RDC sont décrits de différentes manières : 
Tableau 4. Les perceptions à l'égard des pauvres

\begin{tabular}{|c|c|c|}
\hline $\mathbf{N}^{\circ}$ & Perceptions retenues à Kisantu & $\%$ \\
\hline 1 & Sont dépourvus d'argent, de moyens de subsistance et des actifs de vie & 8 \\
\hline 2 & Mangent mal et une fois par jour & 6 \\
\hline 3 & Manquent d'emploi, d'initiative et les paresseux & 6 \\
\hline 4 & Vivent dans un logement indécent et les sans logement & 5 \\
\hline 5 & Sont incapables de résoudre seuls leurs problèmes & 5 \\
\hline 6 & N'ont pas de terres, champs, cheptel, unités de production & 5 \\
\hline 7 & Sont incapables de supporter les études des enfants & 5 \\
\hline 8 & Ont un habillement indécent & 4 \\
\hline 9 & Habitent un Village/Quartier sans écoles & 4 \\
\hline 10 & N'ont pas d'accès à l'eau potable & 3 \\
\hline 11 & Incapables de supporter les coûts de soins médicaux & 3 \\
\hline 12 & $\begin{array}{l}\text { Les maladifs, les handicapés physiques et les gens dont la santé est } \\
\text { précaire }\end{array}$ & 3 \\
\hline 13 & Vivent dans la crainte perpétuelle de la guerre, les gens sans protection & 3 \\
\hline 14 & N’ont pas de matériels de production et d'appui technique & 2 \\
\hline 15 & Déplacés de guerre sans logis & 2 \\
\hline 16 & Les déplacés de guerre & 2 \\
\hline 17 & Habitent un village/Quartier sans structure de santé & 2 \\
\hline 18 & Les enfants en âge scolaire qui ne vont pas à l'école & 2 \\
\hline 19 & Vivent dans un logement sans électricité & 2 \\
\hline 20 & Les orphelins & 2 \\
\hline 21 & Vivent dans des villages dépourvus de services sociaux de base & 2 \\
\hline 22 & Les fonctionnaires et agents de l’Etat mal et non payés & 2 \\
\hline 23 & Les vieux sans appui & 2 \\
\hline 24 & Les analphabètes & 2 \\
\hline 25 & Les familles nombreuses et les femmes surchargées & 1 \\
\hline 26 & Les veuves et femmes seules & 1 \\
\hline 27 & Une famille sans enfant & 1 \\
\hline 28 & Personnes sans revenu, abandonnées par les membres de la famille & 1 \\
\hline 29 & Manquent de moyen de transport personnel (vélo, bicyclette, véhicule,...) & 1 \\
\hline 30 & N'ont pas de frères en ville ou n'ont pas de soutien & 1 \\
\hline & Total & 88 \\
\hline
\end{tabular}

En regroupant ces différentes perceptions qu'ils se sont fait des pauvres, les participants sont arrivés à dégager 9 critères suivants :

- $\quad$ Monétaire 33\%

- $\quad$ Education 13\%

- $\quad$ Santé $10 \%$

- $\quad$ Habitat (logement) 13\%

- $\quad$ Nutrition \& Habillement $10 \%$

- $\quad$ Infrastructures 3\%

- $\quad$ Victimes des conflits 8\%

- $\quad$ Les vulnérables 7\% 
Autres 3\%

Ces critères sont inéluctablement des paramètres de mesure de bien être ou de mal-être. L'analyse du profil et des déterminants de la pauvreté en RDC à travers le DSCRP souligne le rapport d'inter influence entre l'approche monétaire (mesurée sur base du revenu par tête d'habitant) et les autres approches de la pauvreté (pauvreté humaine mesurée à base des capacités de fonctionnement humain, la pauvreté liée aux besoins de base, tels que l'alimentation et le logement).

Au final, toutes ces caractéristiques de la pauvreté proposées par les habitants de la ville de Bukavu et les participants à l'Atelier de Kisantu soulignent globalement que la pauvreté est un tout, rien ne l'explique tout seul et rien n'en dépend tout seul. C'est un cumul de privations. D'ailleurs, même le bien-être ne s'explique pas par un seul fait. Le bonheur devient un objet d'analyse sociologique dès qu'on le constitue comme un fait social, c'est-à-dire qu'on l'explique par d'autres faits sociaux (Aït Saïd Fatima, avril 2011, p.57).

Le manque d'argent est plus évoqué dans les deux camps différents comme sens premier et donc comme première caractéristique de la pauvreté dans la ville de Bukavu et en RDC. Mais une telle approche soulève la question de savoir combien d'argent doit-on manquer ou avoir pour être désigné pauvre ou non pauvre dans le contexte de la ville de Bukavu. Aussi, comme l'affirment Claire Scotton et ses collaborateurs [...] le niveau de vie d'une personne ne dépend pas seulement de ce qu'elle gagne, mais aussi du nombre de personnes avec qui elle vit et de leurs revenus (2005, p. 3). Bien entendu, la question " au-dessous de quel seuil est-on pauvre» est embarrassante, mais comme ailleurs, il s'agit de construire un seuil à partir d'une variable (le revenu médian ou le revenu moyen en général), et de délimiter avec ce seuil une population considérée comme pauvre. Ce choix ne peut être que conventionnel au sein d'une société.

On le voit bien, à Bukavu, celui qui n'a pas d'argent est pauvre, mais aucun repère institutionnel ne permet de dire le montant qu'on doive avoir dans ses actifs pour être défini comme n'étant plus pauvre, ou qu'on l'est encore.

Au demeurant, ces multiples perceptions de la pauvreté font d'elle un spectre et un état dont l'explication, rappelons-le, ne s'appuierait pas sur un monisme ou un déterminisme absolu. Chacune de ces perceptions contribue de telle ou telle façon à la conceptualisation de la pauvreté.

Lorsqu'on rentre dans la littérature sur la pauvreté comme concept, il se dégage qu'elle n'y est pas non plus définie de façon la plus indiscutable, et que bien des aspects de la pauvreté décrits pas les enquêtés à Bukavu ont aussi été effleurés par des scientifiques. 
Ainsi, par exemple pour l'INSEE, la pauvreté est la situation d'une personne (ou d'un ménage) dont le niveau de vie se trouve inférieur au seuil de pauvreté du pays dans lequel il se trouve et l'empêche de vivre une vie normale et de participer aux activités économiques, sociales et culturelles courantes(http://insee.fr/fr/methodes/default.asp?page=definitions/pauvretemonetaire.htm, consulté le 24 juin 2016).

Cette source comme d'autres fait référence à ce dont on peut être privé pour être perçu ou se percevoir comme pauvre. Cette privation est loin d'être seulement matérielle ou financière, mais les critères matériels et financiers semblent être plus mesurables dans l'analyse de la pauvreté.

Nos enquêtés se rallient derrière ce point de vue en évoquant plusieurs privations, partant de celle financière qui, selon eux, détermine les autres privations.

Pour nous, même cette privation financière est déterminée par d'autres privations chez certaines personnes.

Kambamba Darly ajoute pour sa part que les pauvres seraient les personnes dont les ressources restent en-dessous d'un certain seuil (fixé en unité monétaire). Il souligne les répercussions de la pauvreté monétaire sur le social en termes d'incapacité d'une grande majorité des populations congolaises à satisfaire les besoins alimentaires, l’incapacité à accéder aux besoins de santé, à accéder à la scolarisation, à se loger décemment, [...], etc. (mars 2013, consulté sur www.LibreAfrique.org le 09 septembre 2016).

Dans le sillage de cet auteur, un autre analyste de la pauvreté citant le cas du Bénin, revient sur les effets indésirables de la privation monétaire et regrette que des filles n'aient pas eu d'autre choix que de se prostituer à partir de quatorze ans, parfois même de douze, pour un peu d'argent ou simplement pour un dîner (Easterly, W., 2006, p.24). Il se trouve que le manque d'argent entraîne des comportements sociaux de déviance qui deviennent du coup des indicateurs de la pauvreté.

Fort de tout ce qui précède, nous constatons au regard des perceptions de la pauvreté dans la ville de Bukavu que son profil est difficile à présenter mais qu'il s’agit d'un cumul de privations, celle monétaire étant la plus redoutable par les habitants, contre laquelle des mécanismes sont souvent initiés.

La plupart des enquêtés indiquent que ces privations sont corrélées à la pauvreté monétaire, mais il faut souligner, par exemple, que, le passage de l'état de manque d'argent ou de biens à celui d'absence de pouvoir ou d'impossibilité d'en acquérir sur l'ensemble de la société mérite aussi une réflexion profonde.

Il est maintenant important, dans le point suivant, de relever les causes/ les facteurs et les acteurs de la pauvreté dans la ville de Bukavu. 


\section{Analyse De La Causalite Et Des Acteurs De La Pauvrete Dans La Ville De Bukavu \\ Identification des causes/ facteurs de la pauvreté}

Une cause et un facteur ne sont pas tout à fait la même chose. Une cause contribue à l'apparition d'un phénomène, d'un problème ; tandis qu'un facteur contribue à nourrir, alimenter et à faire perdurer ce problème après son apparition(Bartle Phil, 8 juin 2011, consulté sur http://www.versiontracker.com/dyn/moreinfo/macros/2464le 10 août 2016).

Toutefois, dans ce papier, nous ne distinguons pas rigoureusement cause et facteur de la pauvreté dans la ville de Bukavu, pour une raison simple : cause et facteur participent tous au problème. Ce qui nous intéresse ici c'est l'identification d'un ensemble de faits lointains ou proches liés à la pauvreté et par lesquels on l'explique à Bukavu.

Tableau 4. Identification des causes/facteurs de la pauvreté à Bukavu

\begin{tabular}{|c|c|c|c|}
\hline $\mathrm{N}^{\circ}$ & Sources de la pauvreté & Effectif & $\%$ \\
\hline 1 & $\begin{array}{l}\text { Mauvaise gouvernance à tous les niveaux (détournement, enrichissement } \\
\text { illicite, corruption, concussion, etc.) }\end{array}$ & 35 & 10,4 \\
\hline 2 & Manque de volonté politique & 28 & 8,3 \\
\hline 3 & Chômage & 26 & 7,7 \\
\hline 4 & Faiblesse de l’Etat & 25 & 7,4 \\
\hline 5 & Inégalités sociales très poussées & 23 & 6,8 \\
\hline 6 & Pression démographique & 23 & 6,8 \\
\hline 7 & Manque de planification et de projets de société & 21 & 6,2 \\
\hline 8 & Guerres à répétition & 20 & 5,9 \\
\hline 9 & Egoïsme de la part des gouvernants & 19 & 5,6 \\
\hline 10 & Endettement excessif du pays & 17 & 5 \\
\hline 11 & Insuffisance et délabrement d'infrastructures de base & 17 & 5 \\
\hline 12 & Mauvaise application des lois et des décisions politiques & 17 & 5 \\
\hline 14 & Impunité & 15 & 4,4 \\
\hline 15 & Colonisation & 13 & 3,8 \\
\hline 16 & Faible production & 12 & 3,5 \\
\hline 17 & Faible niveau d'étude & 9 & 2,6 \\
\hline 18 & Manque d'esprit d'initiative de la part de certains citoyens & 5 & 1,4 \\
\hline 19 & Paresse de la part de certaines personnes & 5 & 1,4 \\
\hline 20 & La nature & 3 & 0,8 \\
\hline \multirow[t]{2}{*}{21} & Autres & 3 & 0,8 \\
\hline & Total & 336 & 100 \\
\hline
\end{tabular}

La pauvreté vécue dans la ville de Bukavu n'est pas un problème sui generis ; elle tire ses origines dans les faits, les gestes et les discours des hommes, isolés ou agrégés, dans leur rapport avec la société. Elle se propage à la manière d'un virus et s'explique par d'autres phénomènes qui ont pour origine la société.

Ainsi découle-t-il de ce tableau qu'aucune cause ou aucun facteur à lui seul n'explique l'origine de la pauvreté dans la société. L'analyse de la causalité de la pauvreté dans la ville de Bukavu ne peut se cacher derrière le déterminisme, mais requiert une posture plutôt systémique, holiste. Autant 
les significations qu'on lui donne sont multiples et intimement liées, autant les causes ou les facteurs qui l'expliquent sont multiples.

Nos enquêtés semblent se situer dans cette imbrication pauvretéphénomènes économiques et sociaux qui l’alimentent.

Il se dégage que la mauvaise gouvernance et tout ce qu'elle comporte comme effets collatéraux, le manque de volonté politique synonyme de la négligence, de l'insouciance ; le chômage de masse qui empêche l'accès aux revenus; la faiblesse de l'Etat, c'est-à-dire l'insuffisance des capacités extractive, distributive, responsive, régulatrice et symbolique ; la pression démographique et d'autres situations considérées comme entretenues par l'injustice sociale, ne sauraient être ni omis ni dissociés dans la tentative d'explication de la pauvreté à Bukavu.

Dans la ville de Bukavu, les dirigeants et les gestionnaires sont, à tous les niveaux, placés sur le banc des accusés par nos enquêtés. Ils les considèrent comme des égoïstes, travaillant au bénéfice de la pauvreté qu'ils entretiennent par leurs styles de gestion, bien que par leur discours, ils prétendent la combattre. Ils sont perçus comme fonctionnant en dehors de repères éthiques.

Remarquons que certaines de ces causes/facteurs de la pauvreté sont lointains et historiques, d'autres sont immédiats, mais entretiennent des interactions.

A partir de ce qui précède, nous constatons que cette dernière revêt une causalité multiple. Il reste à identifier les instances considérées comme favorisant ou entretenant la pauvreté à Bukavu, mieux, ses acteurs.

\section{Les acteurs de la pauvreté dans la ville de Bukavu}

L'enquêté avait la possibilité de citer plus d'un acteur en répondant à la question « selon vous, quels sont les acteurs de la pauvreté dans la ville de Bukavu » ? C’est ce qui justifie que les données présentées en chiffres dans la figure dépassent l'effectif de 336. 
Figure 1. Les acteurs de la de la pauvreté

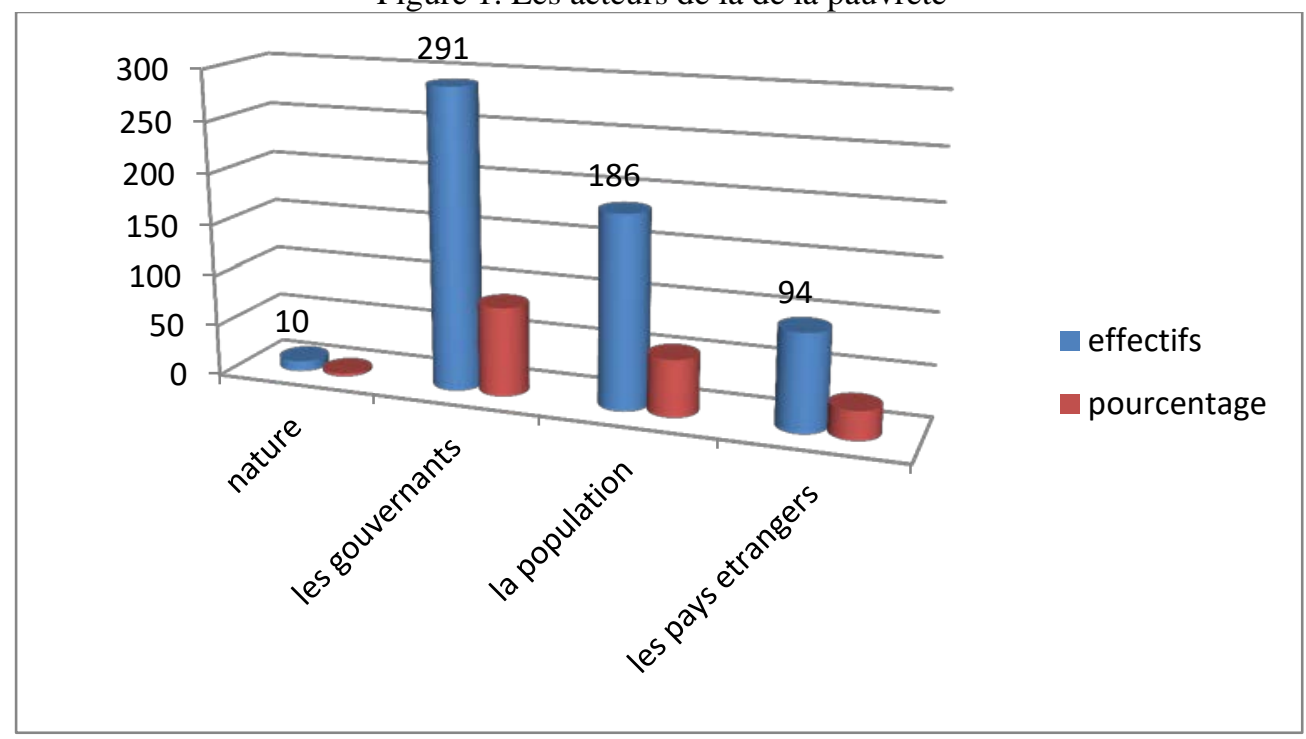

De cette figure, nous retenons que la pauvreté vécue à Bukavu est attribuée à plusieurs acteurs : locaux, étatiques, supra étatiques et la nature. A l'évidence, les gouvernants, chacun en ce qui le concerne, et quel que soit le secteur, suivis de la population dans sa diversité, sont désignés comme les premiers acteurs internes de la pauvreté dans la ville de Bukavu.

Les acteurs agissant de l'extérieur occupent la troisième place dans cette hiérarchie d'acteurs. Quant à la nature, elle est aussi pointée du doigt accusateur, considérant qu'elle est responsable de certaines formes de pauvreté difficile à vaincre par des mécanismes sociaux.

En lisant attentivement le tableau $n^{\circ} 3$ qui précède la figure 01 , on se rend bien compte que chaque cause et facteur de la pauvreté dégagé fait référence à l'un ou l'autre de ces quatre acteurs de la pauvreté ciblés par les enquêtés.

Les motifs pour lesquels chacun de ces acteurs est désigné comme tel ne sont donc pas ignorés par leurs accusateurs. Visiblement, la population connaît les responsabilités de chaque acteur dans la production de ce mal social qu'est la pauvreté.

Et si l'Etat y a sa part de responsabilité, la population aurait aussi contribué au développement de la pauvreté ; comme qui dirait qu'elle a forgé des armes de sa propre destruction. La population passe alors de statut de victime à celui de bourreau, d'opprimé à celui d'oppresseur. C'est notamment par sa passivité interprétée par nos enquêtés comme un indice de complicité ; son égoïsme, ses méthodes irrationnelles de gestion des ressources, sa négligence, etc. 
La pauvreté dans la ville de Bukavu est donc supposée être plus le fruit des erreurs de gestion par les acteurs étatiques et des acteurs non étatiques, situés tous à différents échelons.

Les acteurs d'un autre niveau ont également été désignés, à savoir les pays étrangers ou les acteurs internationaux.

Les enquêtés sont convaincus que les pays étrangers contribuent à la pauvreté en agissant activement à travers:

- $\quad$ l'exploitation ou le pillage des ressources naturelles de la RDC ;

- $\quad$ le soutien des actions belliqueuses en RDC (soutien des guerres d'agression);

- $\quad$ En matière de commerce, les puissances étrangères imposent à la RDC l'importation au lieu d'encourager l'exportation ;

- $\quad$ L'interférence dans les affaires intérieures de la RDC et l'imposition d'une vision dans le choix des dirigeants ;

- $\quad$ L'insuffisante implantation d'entreprises à Bukavu pour créer des emplois aux populations locales ;

- $\quad$ La poursuite de la colonisation par des tactiques habiles et douces.

Ces considérations laissent comprendre que le phénomène de pauvreté peut être local tout en ayant certaines explications de niveau global. Les acteurs internationaux sont en interactions diverses avec les acteurs locaux et nationaux agissant au niveau interne.

Quant à la nature, bien qu'ayant été évoquée parmi les acteurs de la pauvreté, elle n'est pas du tout un acteur aussi considérable que les acteurs précédant. Certains l'ont même considérée comme une des causes de la pauvreté. Pourtant, à Bukavu, on est convaincu que la pauvreté est un état, on peut y entrer à un moment donné, en sortir à un autre moment et s'y réintégrer éventuellement. C’est donc essentiellement une affaire de la société que de la nature.

A partir de ces résultats, il n'est pas superflu de penser que la pauvreté, appréhendée dans une vision à la fois systémique et dialectique, procède de contradictions au niveau discursif et praxéologique entre différents acteurs tant locaux que nationaux et supranationaux en interdépendance.

\section{Conclusion}

En identifiant la signification que les habitants de la ville de Bukavu donnent à la pauvreté, les causes ou les facteurs à son origine et les acteurs accusés comme travaillant consciemment ou inconsciemment pour sa pérennisation, cette étude confirme l'existence de la pauvreté et des pauvres dans cette entité. 
Il va de soi que dans la ville de Bukavu, la pauvreté demeure à la fois un discours et une réalité. Elle est vécue, observée, décrite, mais dire ce qu'elle signifie continue à être malaisé, même par ceux qui s'en plaignent.

Bien plus, la définir par un monisme le plus absolu serait une erreur, au regard de sa multi dimensionnalité.

Il est ressorti de cette étude que sur les plans factuel et discursif, la pauvreté est observable et se propage à Bukavu. Elle est reconnue par ses manifestations extérieures qui s'offrent à l'observation. Ses causes / facteurs sont de plus en plus multiples autant que les sens qui lui sont attribués.

Ainsi retenons-nous de cette recherche que la pauvreté reste un cumul de privations, de manques, non seulement concernant les aspects économiques et financiers les plus sensibles de la vie, mais également ceux qui touchent au social. Les résultats de l'enquête renseignent cependant que pour les habitants de la ville de Bukavu, la pauvreté est surtout une situation où on est privé de l'argent de façon permanente. Pour eux, le manque d'argent entraîne d'autres multiples privations, et inversement, la ressource argent procure d'autres ressources non matérielles. Ce manque d'argent est lié entre autres au chômage, à la gouvernance jugée mauvaise, au manque d'accès aux opportunités, etc. C'est là la complexité du phénomène de pauvreté. Rien ne l'explique tout seul, elle est en interaction avec les autres phénomènes de la société qui l'expliquent ou qu'elle explique.

Compte tenu de ces considérations, le dressement du portrait de la pauvreté dans la ville de Bukavu ne pourrait relever que d'un essai à actualiser au quotidien, en intégrant plusieurs indicateurs à la fois monétaires et sociaux agrégés. S’il y a de consensus sur son existence, il faut cependant rester prudent sur ce qu'elle signifie et sur ce qui la provoque, en dépit du fait que l'aspect "argent" soit magnifié dans l'appréhension de la pauvreté et de la non pauvreté à Bukavu.

\section{References:}

1. Aït Saïd Fatima, (avril 2011). «Le bonheur en économie », Idées Economiques et sociales, $\mathrm{n}^{\circ} 166$.

2. Anita Kirpalani, (25 avril 2013). «Indice du progrès social: nouvel instrument pour mesurer le bien-être ", Youphil.com, le média de toutes les solidarités.

3. Banque mondiale, (2000-2001). Rapport sur le développement dans le monde, Combattre la pauvreté.

4. Bartle, P., (2007). « Facteurs de la pauvreté. Les cinq plaies », sé. mis à jour le 8juin2011, consulté surhttp://www.versiontracker.com/dyn/moreinfo/macros/2464, le 10 août 2016. 
5. DSCRP, (2006), Profil et déterminants de la pauvreté en RDC : ce que le DSCRP promet de corriger,consulté sur http : //www.dscrprdc.org/ le 4mai 2016

6. http://insee.fr/fr/methodes/default.asp?page=definitions/pauvretemonetaire.htm

7. Kambamba Darly, (18 mars 2013). «RDC : Pourquoi tant de pauvreté ? ", in Libre Afrique. Un regard alternatif sur le continent, consulté sur www.LibreAfrique.org le 09 mai 2016.

8. PNUD, (2013). Taux de pauvreté par province en RDC.

9. RDC, (2005). Rapport sur le diagnostic de la pauvreté en RDC.

10. Scotton, C., Villemonteix, T. et Gaini, M., (2005). « Mesure et Démesure de la pauvreté en France : Qui sont les pauvres ? Quelles politiques contre la pauvreté »,sl.

11. William Easterly, (2006). Les pays pauvres sont-ils condamnés à le rester ? Paris, Nouveaux Horizons, Groupe Eyrolles. 